\title{
Influence of multi-walled carbon nanotubes on the cognitive abilities of Wistar rats
}

\author{
NINA V. SAYAPINA ${ }^{1}$, ALEXANDER A. SERGIEVICH ${ }^{2}$, VLADIMIR L. KUZNETSOV ${ }^{3}$, VLADIMIR V.CHAIKA ${ }^{2}$, \\ IRINA G. LISITSKAYA ${ }^{2}$, PAVEL P. KHOROSHIKH ${ }^{4}$, TATYANA A. BATALOVA ${ }^{1}$, KOSTAS TSAROUHAS ${ }^{5}$, \\ DEMETRIOS SPANDIDOS $^{6}$, ARISTIDIS M. TSATSAKIS ${ }^{2,7}$, CONCETTINA FENGA $^{8}$ and KIRILL S. GOLOKHVAST ${ }^{2}$ \\ ${ }^{1}$ Department of Physiology, Amur State Medical Academy, 675000 Blagoveshchensk; \\ ${ }^{2}$ Scientific Educational Center of Nanotechnology, Far Eastern Federal University, 690950 Vladivostok; \\ ${ }^{3}$ Group of Surface Compounds Synthesis, Boreskov Institute of Catalysis SB RAS, 630090 Novosibirsk; \\ ${ }^{4}$ Technical Institute, North-Eastern Federal University, 678962 Nerungri, Russia; ${ }^{5}$ Cardiology Department, \\ University Hospital of Larissa, 41110 Thessaly; ${ }^{6}$ Laboratory of Clinical Virology; \\ ${ }^{7}$ Department of Forensic Sciences and Toxicology, Medical School, University of Crete, 74100 Heraklion, \\ Greece; ${ }^{8}$ Department of Occupational Medicine, University of Messina, 98166 Messina, Italy
}

Received February 10, 2016; Accepted May 17, 2016

DOI: $10.3892 /$ etm.2016.3495

\begin{abstract}
Studies of the neurobehavioral effects of carbon nanomaterials, particularly those of multi-walled carbon nanotubes (MWCNTs), have concentrated on cognitive effects, but data are scarce. The aim of this study was to assess the influence of MWCNTs on a number of higher nervous system functions of Wistar rats. For a period of 10 days, two experimental groups were fed with MWCNTs of different diameters (MWCNT-1 group, 8-10 nm; MWCNT-2 group, 18-20 nm) once a day at a dosage of $500 \mathrm{mg} / \mathrm{kg}$. In the open-field test, reductions of integral indications of researching activity were observed for the two MWCNT-treated groups, with a parallel significant $(\mathrm{P}<0.01)$ increase in stress levels for these groups compared with the untreated control group. In the elevated plus-maze test, integral indices of researching activity in the MWCNT-1 and MWCNT-2 groups reduced by day 10 by 51 and $62 \%$, respectively, while rat stress levels remained relatively unchanged. In the universal problem solving box test, reductions in motivation and energy indices of researching activity were observed in the two experimental groups. Searching activity in the MWCNT-1 group by day 3 was reduced by $50 \%(\mathrm{P}<0.01)$ and in the MWCNT-2 group the relevant reduction reached $11.2 \%$. By day 10, the reduction compared with controls, was $64 \%$ $(\mathrm{P}<0.01)$ and 58\% $(\mathrm{P}<0.01)$ for the MWCNT-1 and MWCNT-2
\end{abstract}

Correspondence to: Professor Kirill Sergeevich Golokhvast, Scientific Educational Center of Nanotechnology, Far Eastern Federal University, 10 Pushkinskaya Street, 690950 Vladivostok, Russia

E-mail: droopy@mail.ru

Key words: cognitive, stress, multi-walled carbon nanotubes, Wistar rats groups, respectively. In conclusion, a series of specific tests demonstrated that MWCNT-treated rats experienced a significant reduction of some of their cognitive abilities, a disturbing and worrying finding, taking into consideration the continuing and accelerating use of carbon nanotubes in medicine and science.

\section{Introduction}

New materials are appearing in the world of modern technology, with nanomaterials, such as nanotubes, nanoballs, nanorods, quantum dots, immobilized nanoparticles and a number of other unusual structures, undergoing the most development and being particularly noteworthy (1).

The volume of nanomaterial production has increased in volume from early laboratory samples delivered in microgram quantities to current multi-ton production. As a result, their presence in people's everyday lives and the environmental burden they may cause is constantly increasing (1).

Knowledge concerning the toxicological properties of carbon nanomaterials in general and nanotubes in particular is evolving. Nanotubes are one- or multi-walled cylindrical structures, made from carbon (2). Information on nanotubes' toxicity has appeared in the world literature (3) since the early 2000 s, and research in this field is ongoing (3). Carbon nanotubes are considered to be rather toxic due to their ability to cause apoptosis (4) and demonstrate mutagenic properties as a result of increasing the formation of reactive oxygen species (5). Biodegradation of nanotubes has been recorded (6).

One of the most important concerns is neurotoxicity of carbon nanomaterials, as they are considered to cause a series of neurodegenerative disorders, mainly due to oxidative stress accumulation and a parallel reduction in antioxidant protection mechanisms $(7,8)$.

Investigation of the neurobehavioral aspects of the effects of carbon nanomaterials, especially those of multi-walled 
carbon nanotubes (MWCNTs), has recently concentrated on cognitive effects (3), but data are scarce. Recent studies suggest that nanotube-induced neurotoxicity may result from a local increase in the level of oxidative stress in the hippocampal region of the brain. The first histological confirmation of inflammation in said region of the brain has been provided, along with evidence of altered oxidative stress parameters (7-9).

However, studies have demonstrated that nanotube diameter is crucial to their toxicity, as thinner MWCNTs both in vitro and in vivo appeared significantly more toxic than thicker ones in rat studies of lung toxicity $(10,11)$.

The aim of the present study was to assess the influence of MWCNTs on a number of indicators of higher nervous system activity in Wistar rats.

\section{Materials and methods}

Animals. Male Wistar rats, weighing 100-200 g, were housed in the Animal Center of Amur State Medical Academy (Blagoveshchensk, Russia). They were kept under standard conditions $\left(21-23^{\circ} \mathrm{C}\right.$ temperature, $12 \mathrm{~h} / 12 \mathrm{~h}$ light/dark cycle) with free access to food and water. They were housed in spacious cages, separate from other species.

All animals were treated in accordance with the European Guidelines for the Care and Use of Laboratory Animals. The study was approved by the Biomedical Ethical Committee of Amur State Medical Academy.

Experimental groups and study design. A total of 30 Wistar rats aged 3 months and weighing $180 \pm 200 \mathrm{~g}$ took part in the study. The study groups comprised two experimental groups and a control group. Animals were randomly assigned to the three groups. For a period of 10 days, once a day, the two experimental groups were given MWCNTs as nutrition at a dosage of $500 \mathrm{mg} / \mathrm{kg}$. The amount of MWCNT per animal was mixed with a base comprising $5 \mathrm{~g}$ feed (3-grain; Fermic, Iztapalapa, Mexico) and raw eggs. The control group was given the feed base without any amount of MWCNT. A number of experiments were included in the study scheme and were carried out on days 1, 3 and 10 of the 10-day experimental period.

Characteristics of nanomaterials. The first experimental group received MWCNTs (MWCNT-1) with a diameter of $8-10 \mathrm{~nm}$ and specific surface of $400 \mathrm{~m}^{2} / \mathrm{g}$, and the coexisting impurities in its structure were $0.6 \% \mathrm{Fe}, 0.3 \% \mathrm{Co}$ and $0.9 \% \mathrm{Al}$. Open field testing data obtained for MWCNT-1 by our group has previously been published (12). The second experimental group received MWCNTs (MWCNT-2) with a diameter of $18-20 \mathrm{~nm}$ and specific surface of $130 \mathrm{~m}^{2} / \mathrm{g}$, and the coexisting impurities were $0.2 \% \mathrm{Fe}, 0.12 \% \mathrm{Co}, 0,004 \% \mathrm{Ca}$ and $0.08 \%$ $\mathrm{Cl}$ in its structure. The two types of MWCNT represented aggregates with sizes from 5-100 $\mu \mathrm{m}$.

Characterization of the materials and their structure was performed using an S-3400N scanning electron microscope (Hitachi, Ltd., Tokyo, Japan) and Ultra Dry energy dispersive spectrometer (Thermo Fisher Scientific, Inc., Waltham, MA, USA). Metal impurities were assessed using X-ray fluorescence analysis (XrFA). The wave dispersive X-ray fluorescence spectrometer ARL Advant'x 3600 (Thermo
Fisher Scientific, Inc.) was used to conduct the XrFA of the MWCNTs. Instrumental control was possible with the use of OXSAS software, version 1.5.

The S-3400N scanning electron microscope and the Ultra Dry spectrometer were used for assessment of the nanotube aggregates.

Physiological evaluation. All animals from the two experimental groups and the control group were submitted to physiological evaluation through the use of an open field test, elevated plus-maze test and universal problem solving test. Animal training and testing experiments were performed during the daytime. The open-field test (13) and elevated plus-maze test (14) are well-known in behavioral physiology as objective assessments for obtaining accurate data on individual stress and researching activity. The universal problem-solving box (UPSB) of Grigor'ev et al (15) was used to analyze alterations in the finding-activity dynamics of treated animals due to the oral intake of carbon nanotubes. A detailed description of the tests are as follows:

Open-field test. The open-field test is a test with proven efficacy for the evaluation of laboratory rats in terms of judging the elements of a difficult behavioral act in moderate stress conditions (13). The emotional status of the animals can be recorded, bearing in mind that the animal is housed in new conditions, which promotes animal stress.

The test was performed under standard lighting, and at the same time of day (16:00). Open-field areas were 100x100-cm wide, and were divided into 25 squares with 16 holes located at the intersection of the squares (Fig. 1). Tested animals were placed individually in the center of the open-field as the starting place; the starting place was $20 \times 20 \mathrm{~cm}$ wide. Video cameras traced the rat movements over a 3-min period. After each animal examination the surface of the open-field was cleaned with fresh water several times and dried.

During the test a number of different types of activity were assessed, all associated with the researching behavior response (16): i) Spontaneous researching activity/explorative activity, shown as the number of holes explored by the animals; ii) orientative activity, shown as the number of vertical rears and iii) locomotor activity, assessed as the number of crossings of internal and external squares. The latency time of exiting the center and time of general activity were also measured. An integral estimate of researching behavior was determined as the sum of the researching behaviour scores and the stress level scores.

Emotional status was represented by the level of experimental rat stress and was assessed by following variables: i) The general time of immobility (freezing), ii) the number of grooming acts and iii) defecation activity (the number of boluses) (15). The sum of the points for all the above variables formed the stress level (15).

Elevated plus-maze test. The elevated plus-maze test $(17,18)$, in addition to the open-field test, is known to be an efficient method of assessing the activities and stress of small animals. The method is based on the inborn rat's fear of heights, open areas and its natural researching behavior, which appears when a new environment is encountered by the animal.

The elevated plus-maze test comprises a system of open and closed sleeves with a height of $77 \mathrm{~cm}$ and length of $90 \mathrm{~cm}$ 


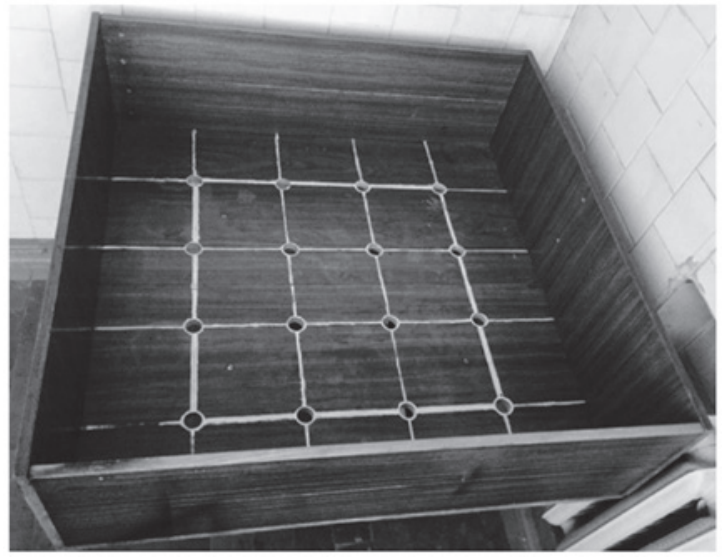

Figure 1. Open field test.

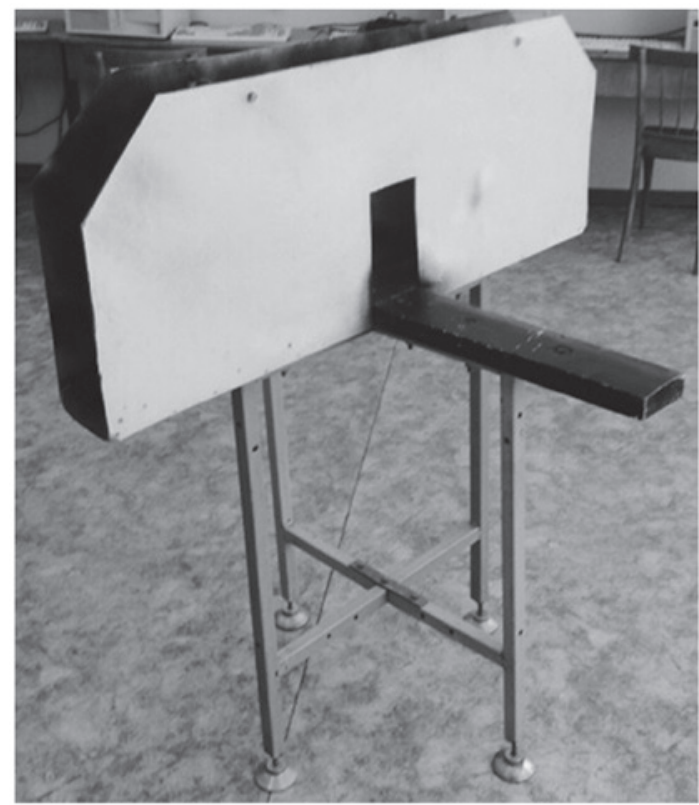

Figure 2. Elevated plus-maze test.

(Fig. 2). The Wistar rat was placed in the center of the apparatus and all the movements of the rat were traced using video cameras. Each animal was tested in the maze for a 3-min time period.

The level of individual stress of each Wistar rat was assessed (15) according to the following indices: i) Time spent in the closed sleeves and ii) the number of grooming acts and boluses. Researching activity was assessed by the following indices: i) Time spent in the open sleeves, ii) time of movement activity and iii) the number of vertical standing and bending activities.

UPSB test. Searching activities of the rats and peculiarities of information and emotional stress were studied through the use of a UPSB (Fig. 3) (19,20). The UPSB is a hexagonal pyramid with a transparent cover and a hatch for the rats to enter. The side walls of the pyramid have six outlets with transparent suspended doors that are monitored with the help of sensors in order to record any door touching by the animals.

The UPSB can explore the possibility of a cognitive aim by the rats, which is represented by the finding of the correct

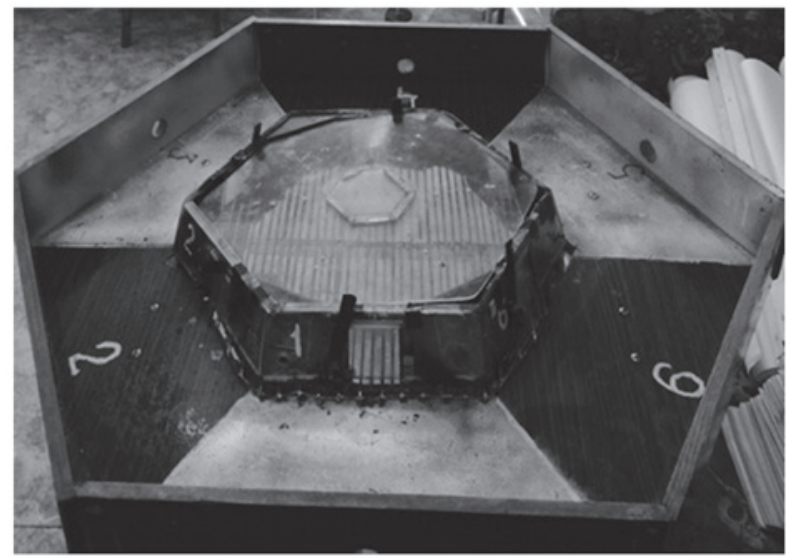

Figure 3. Universal problem-solving box.

box door, in this case to satisfy food needs. One of the challenges of the test is that with every new run the doors are locked so the researching aim becomes more difficult. The rat has to decide where to run. Excursions were considered to be correct when the rat used a previously unused door, and erroneous when directed to a locked door. The sum of correct or contrary incorrect rat decisions was dependent upon each rat's cognitive ability. The specificity of the test method has been previously described (15). The method permits the registration and assessment of rat finding activity based on informational-emotional stress.

Researching activity was assessed in the structure of feeding behavior (on the basis of formed instrumental feed-finding reflexive behaviour) and represents a safe method for assessing the cognitive abilities of laboratory rats.

Prior to the experimental scheme and on 3 days all rats underwent preliminary preparation that included familiarization with the problem-solving box and conditioning of instrumental feed-finding reflect. Following the preparatory period and the initiation of the experimental scheme, all tests were carried out on days 1, 3 and 10 of the experiment.

Statistical analysis. Data analysis was performed with the use of the Statistica 6.0 statistical software package (StatSoft; Dell Software, Round Rock, TX, USA). Data are expressed as mean \pm standard deviation. Comparative analyses were conducted using Student's t-tests and Mann-Whitney U tests. Comparative analysis of orientative-researching activity and stress levels between experimental groups and in comparison to the control group were conducted using the Mann-Whitney $\mathrm{U}$ test. Differences were considered significant at $\mathrm{P}<0.05$.

\section{Results}

Open-field test. In the open-field test a reduction of researching activity in the treated rats along with an increase of rat stress were recorded. Table I summarizes the findings of the open-field test for orientation researching activity.

Analysis of the orientation-researching activity components revealed that in the MWCNT-1 group, the general time of moving activity was reduced on day 3 by $45 \%$ compared with the day 1 measurement, and by day 10 , the reduction reached $79 \%(\mathrm{P}<0.01)$. A changing dynamic of internal square 
crossings in the MWCNT-1 group was evident on day 10 of the experiment and was reduced by $58 \%$ compared with the day 1 measurement $(\mathrm{P}<0.001)$. A reduction of the number of external square crossings was observed on days 3 and 10 , and the reduction was 44 and $73 \%(\mathrm{P}<0.01)$, respectively. A change of spontaneous researching activity, exhibited as searching of the holes, was found on day 10 with a reduction of $43 \%$ $(\mathrm{P}<0.05)$. The number of vertical standings reduced on all days of the experimental test, and by days 3 and 10 the reduction was 73 and $87 \%(\mathrm{P}<0.01)$, respectively.

In the MWCNT-2 group the general time of moving activity was reduced on days 3 and 10 by $74 \%(\mathrm{P}<0.05)$ and $77 \%$ $(\mathrm{P}<0.05)$, respectively. The number of internal square crossings of the field was reduced on days 3 and 10 by $58 \%(\mathrm{P}<0.05)$ and $74 \%(\mathrm{P}<0.01)$, respectively. The number of external square crossings was reduced by $75 \%(\mathrm{P}<0.01)$ on day 3 and by $84 \%$ $(\mathrm{P}<0.01)$ on day 10 . Reductions of vertical standings were 82 and $78 \%(\mathrm{P}<0.01)$ on days 3 and 10 , respectively.

Changes of integral indications of researching activity in the open-field test were observed (Fig. 4). The Mann-Whitney test showed a significant change of integral indications in the MWCNT-1 group on day 10 and in the MWCNT-2 group on days 3 and 10 .

An increase of the general time of immobility was observed on days 3 and 10 by 133.1 and $95.6 \%(\mathrm{P}<0.01)$ and by 83.4 and $101.3 \%$ in the MWCNT-1 and MWCNT-2 groups, respectively.

The change of emotional status in the open-field test is presented in Fig. 5. A significant change of the Wistar rats' stress levels $(\mathrm{P}<0.01)$ was observed from day 10 measurements for both groups.

Elevated plus-maze test. Elevated plus-maze test results revealed a reduction of researching activity indicators. In the MWCNT-1 group on day 10, time spent in the open sleeve was reduced by $27 \%(\mathrm{P}>0.05)$, time of moving activity was reduced by $65 \%(\mathrm{P}<0.05)$, the number of bendings by $58 \%(\mathrm{P}<0.05)$ and vertical standings by $49 \%(\mathrm{P}<0.05)$. In the MWCNT-2 group on day 10, the time spent in the open sleeves was reduced by $57 \%$ ( $\mathrm{P}>0.05)$, time of moving activity reduced by $70 \%(\mathrm{P}<0.01)$, number of bendings reduced by $68 \%(\mathrm{P}<0.01)$ and the number of vertical standings reduced by $47 \%(\mathrm{P}<0.05$; Table II).

Integral indices of researching activity in the MWCNT-1 and MWCNT- 2 groups reduced on day 10 by 51 and $62 \%$ respectively (Fig. 6).

On day 10, the stress level had increased in the MWCNT-1 group by $3 \%$ and in the MWCNT-2 group by only by $0.7 \%$. It is evident that the stress levels in the experimental groups presented no alterations compared with starting levels. Compared with the control group, both experimental groups presented significant differences in measurements of stress levels on day 10 ( $\mathrm{P}<0.05$; Fig. 7).

Summarizing the above results from the elevated plus-maze test, it is evident that oral administration of carbon nanotubes of different diameters in the standard dose of $500 \mathrm{mg} / \mathrm{kg}$ reduced the indices of researching activity, while rat stress levels remained relatively constant.

UPSB test. Analysis of the results for the UPSB test revealed a reduction in motivation and energy indices of researching 

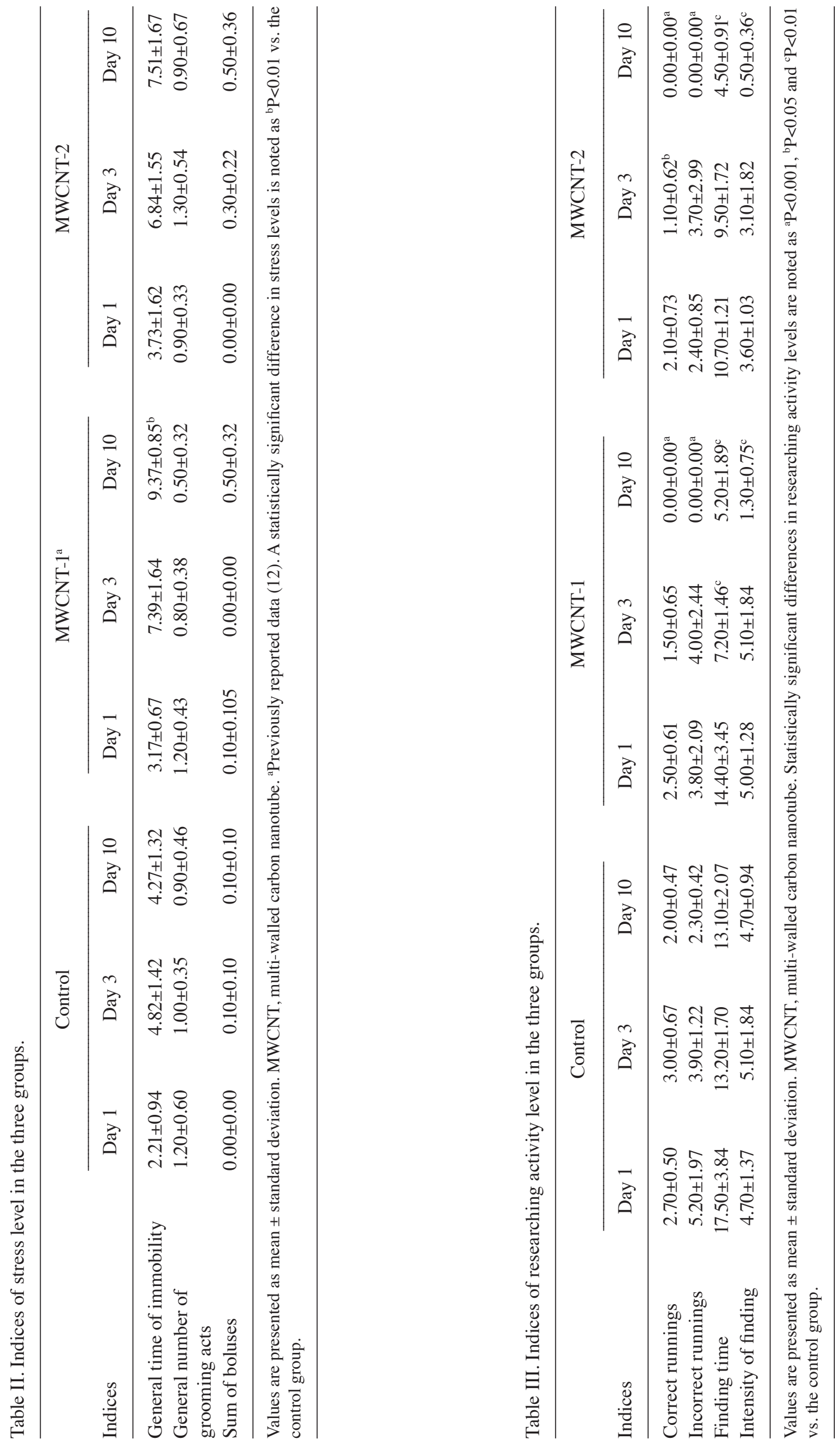
Table IV. Significance of changes in researching behavior of Wistar rats in the UPSB test.

\begin{tabular}{lrl}
\hline Group & Day & P-value \\
\hline MWCNT-1 & 1 & 0.88 \\
& 3 & 0.096 \\
MWCNT-2 & 10 & 0.001 \\
& 1 & 0.88 \\
& 3 & 0.06 \\
& 10 & 0.001 \\
\hline
\end{tabular}

P-values vs. control determined by Mann-Whitney U test. UPSB, universal problem-solving box; MWCNT, multi-walled carbon nanotube.

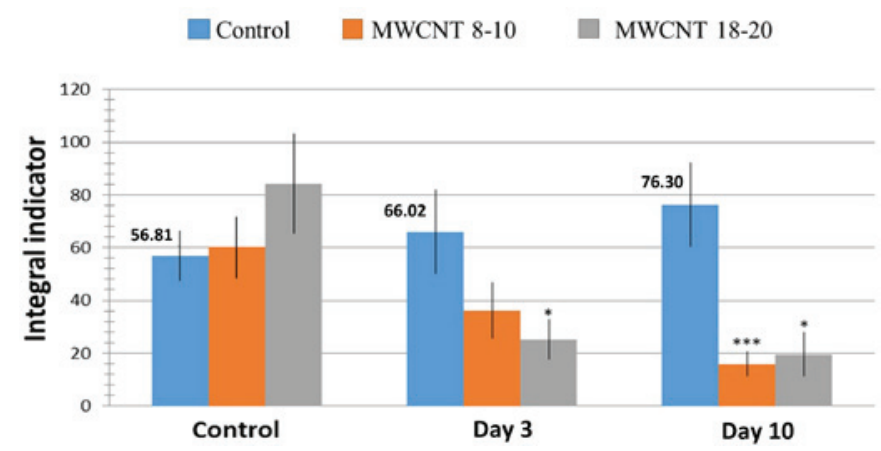

Figure 4. Changes of integral indications of researching activity in the open-field test. Data are presented as the mean \pm standard deviation. ${ }^{*} \mathrm{P}<0.05$ and ${ }^{* * *} \mathrm{P}<0.001$ vs. the control. MWCNT, multi-walled carbon nanotube.

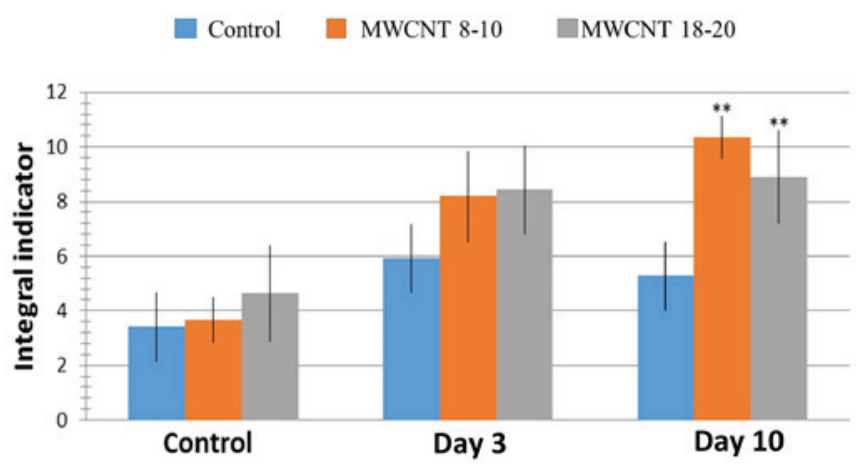

Figure 5. Changes in the emotional status of rats in the open-field test. Data are presented as the mean \pm standard deviation. ${ }^{* *} \mathrm{P}<0.01$ vs. the control. MWCNT, multi-walled carbon nanotube.

activity in the experimental groups. Searching activity in the MWCNT-1 group by day 3 reduced by $50 \%(\mathrm{P}<0.01)$ and in the MWCNT-2 group said reduction was $11.2 \%$. By day 10 , in comparison with control group values the reduction was $64 \%$ and 58\% (both $\mathrm{P}<0.01$ ) for the MWCNT-1 and MWCNT-2 groups, respectively.

Dynamics of active researching were reduced on the basis of the day 10 measurements. In the MWCNT-1 group the reduction was $74 \%(\mathrm{P}<0.01)$ and in the MWCNT-2 group it was $86.1 \%(\mathrm{P}<0.01)$.

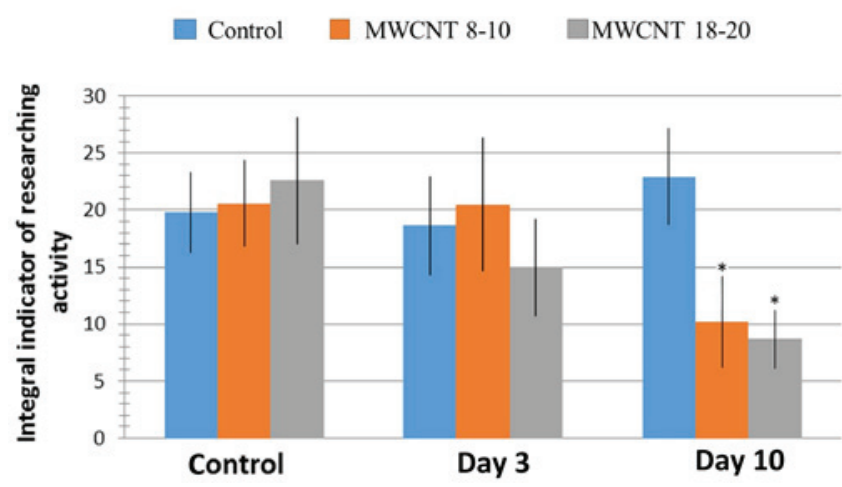

Figure 6. Changes of integral indices of researching activity in the elevated plus-maze test. Data are presented as the mean \pm standard deviation. "P $<0.05$ vs. the control. MWCNT, multi-walled carbon nanotube.

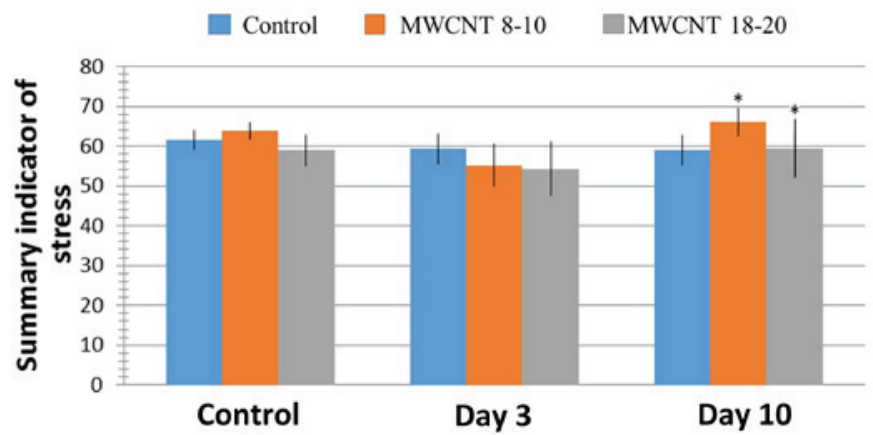

Figure 7. Changes of stress levels in the elevated plus-maze test. Data are presented as the mean \pm standard deviation. ${ }^{*} \mathrm{P}<0.05$ vs. the control. MWCNT, multi-walled carbon nanotube.

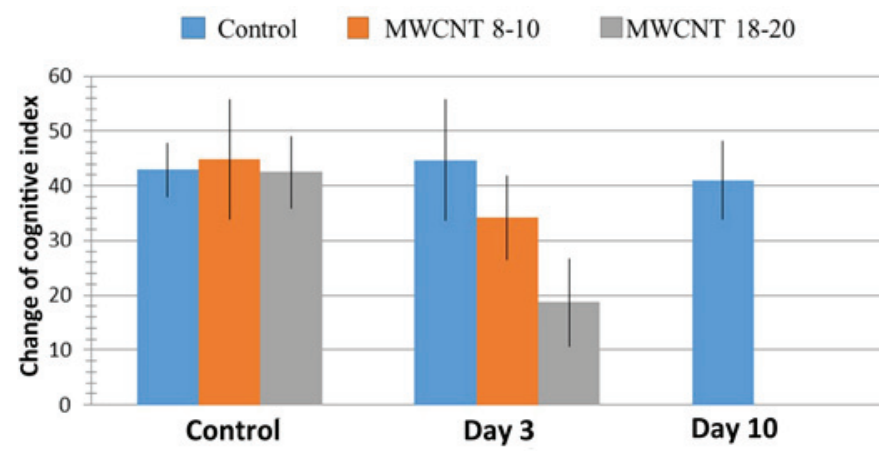

Figure 8. Changes of cognitive index in Grigoriev's universal problem-solving box test. Data are presented as the mean \pm standard deviation. MWCNT, multi-walled carbon nanotube.

In addition, a strong tendency of the MWCNT-2 group rats for a reduction in the number of correct runnings and increase in the number of incorrect ones was observed in the test. From the day 10 measurements, an absolute refusal to research was observed in the two experimental groups (Table III).

Changes in cognitive index during the 10-day experimental period are shown in Fig. 8. Assessing the researching behavior of the Wistar rats through the UPSB test, it was observed that a 10-day scheme of oral administration of carbon nanotubes with different diameters led to the reduction of motivation and energy indices of researching activity and cognitive 
abilities (Table IV). By day 10, treatment of the Wistar rats with MWCNTs had completely abolished their researching activity.

\section{Discussion}

Carbon nanotubes with their broad spectrum of applications in biomedicine, especially in the field of neuroscience as drug carriers (21) or in electrical nerve stimulation (3), create a requirement for close surveillance and monitoring of potential toxicity. To the authors' knowledge, there are very few in vivo studies of neurotoxicity either for single-wall carbon nanotubes (8) or MWCNTs (3). In the present study, the behavioral and cognitive effects on rats of MWCNTs with different diameters were studied. The results revealed a marked tendency of MWCNT-exposed rats to depressed behavior, a parallel reduction of cognitive abilities and an increase of stress levels.

In a previous study on cognitive disability due to MWCNT administration (3), the memory and learning ability of MWCNT-treated rats was affected, according to Morris water maze test results. In the present study, a series of different tests were used in order to investigate rat cognitive ability, while animal stress was recorded. The results of the present study are in agreement with those of Gao et al (3), as MWCNT-treated rats presented reduced cognitive ability. The oral administration route was selected in the present study in contrast to previous studies $(3,8)$. In the case of oral administration, bioabsorption depends on the characteristics of the substance, the physiological environment of the gastrointestinal tract and food intake (22). This usually leads to a lower bioabsorption than that achieved with the usual intraperitoneal or intravenous administration.

Despite previous results showing that MWCNTs with a smaller diameter have greater toxicity $(10,11)$, the animals treated with higher diameter MWCNTs in the present study showed an equally significant decline of cognitive ability in all the tests, with the absolute values being rather worse than those from the animals treated with the MWCNTs having a lower diameter.

The open-field test and UPSB test showed an increase of rat stress levels, while in the elevated plus-maze test no changes in the rat stress levels were recorded. This observation can be attributed to the different experimental test conditions. It could be suggested that the closed sleeves in the elevated plus-maze test allow the rat to hide and this is the reason of the observed steady stress levels.

Increased stress has a negative effect on researching activity; however, the development of local brain inflammation due to MWCNT exposure could also account for the development of cognitive disability, as new data have indicated $(8,23)$. Mechanistically, inflammation alters monoamine levels leading to cognitive and affective dysfunction (24). However, researching activity differs during testing due to high sensitivity to external factors. A recent study demonstrated that unstable rats differ in researching activity and behavioral depression (25).

Moreover, researching activity in neurophysiology is associated with the activity of adreno-positive substances, as hippocampal structures of the brain are very sensitive to such substances leading to neurophysiological damage of catecholaminergic systems. It can be suggested that oxidative damage in the rat hippocampus may be one of the reasons for the observed reduction in researching activity and cognitive disability of rats exposed to nanotubes (8). This phenomenon can be connected to mechanisms of memory violation, which are the most important components of cognitive behavior. However, when the rat does not try to find any exit out of the stress conditions, refusal behavior is observed, which could be a reason accounting for the suppression of researching activity in the open-field test and in the elevated plus-maze test.

In conclusion, cognitive impairment induced by MWCNT exposure of Wistar rats raises concerns about the use of carbon nanotubes, especially MWCNTs, particularly when their increasing applications in science and medicine is considered. Determination of the mechanism of toxicity, judgment of specific toxicity levels through a detailed risk assessment and establishment of potential protective measures through future studies could help to enable the safe and unimpeded use of all classes of carbon nanotubes.

\section{Acknowledgements}

This study was supported by the Russian Scientific Foundation (no. 15-14-20032).

\section{References}

1. Popp L and Segatori L: Differential autophagic responses to nano-sized materials. Curr Opin Biotechnol 36: 129-136, 2015.

2. Wang CY, Zhang YY, Wang CM and Tan VB: Buckling of carbon nanotubes: A literature survey. J Nanosci Nanotechnol 7: 4221-4247, 2007.

3. Gao J, Zhang X, Yu M, Ren G and Yang Z: Cognitive deficits induced by multi-walled carbon nanotubes via the autophagic pathway. Toxicology 337: 21-29, 2015.

4. Bottini M, Bruckner S, Nika K, Bottini N, Bellucci S, Magrini A, Bergamaschi A and Mustelin T: Multi-walled carbon nanotubes induce T lymphocyte apoptosis. Toxicol Lett 160: 121-126, 2006.

5. Møller P, Jensen DM, Christophersen DV, Kermanizadeh A, Jacobsen NR, Hemmingsen JG, Danielsen PH, Karottki DG, Roursgaard M, Cao Y, et al: Measurement of oxidative damage to DNA in nanomaterial exposed cells and animals. Environ Mol Mutagen 56: 97-110, 2015

6. Elgrabli D, Dachraoui W, Ménard-Moyon C, Liu XJ, Bégin D, Bégin-Colin S, Bianco A, Gazeau F and Alloyeau D: Carbon nanotube degradation in macrophages: Live nanoscale monitoring and understanding of biological pathway. ACS Nano 9: 10113-10124, 2015

7. Dal Bosco L, Weber GE, Parfitt GM, Paese K, Gonçalves CO, Serodre TM, Furtado CA, Santos AP, Monserrat JM and Barros DM: PEGylated carbon nanotubes impair retrieval of contextual fear memory and alter oxidative stress parameters in the rat hippocampus. Biomed Res Int 2015: 104135, 2015.

8. Liu X, Zhang Y, Li J, Wang D, Wu Y, Li Y, Lu Z, Yu SC, Li R and Yang X: Cognitive deficits and decreased locomotor activity induced by single-walled carbon nanotubes and neuroprotective effects of ascorbic acid. Int J Nanomedicine 9: 823-839, 2014.

9. Ivani S, Karimi I, Tabatabaei SR and Syedmoradi L: Effects of prenatal exposure to single-wall carbon nanotubes on reproductive performance and neurodevelopment in mice. Toxicol Ind Health: 0748233714555388, 2014.

10. Fenoglio I, Aldieri E, Gazzano E, Cesano F, Colonna M, Scarano D, Mazzucco G, Attanasio A, Yakoub Y, Lison D and Fubini B: Thickness of multiwalled carbon nanotubes affects their lung toxicity. Chem Res Toxicol 25: 74-82, 2012.

11. Nagai H, Okazaki Y, Chew SH, Misawa N, Yamashita Y, Akatsuka S, Ishihara T, Yamashita K, Yoshikawa Y, Yasui H, et al: Diameter and rigidity of multiwalled carbon nanotubes are critical factors in mesothelial injury and carcinogenesis. Proc Natl Acad Sci USA 108: E1330-E1338, 2011. 
12. Sayapina NV, Batalova TA, Chaika VV, Kuznetsov VL Sergievich AA, Kolosov VP, Perel'man YM and Golokhvast KS: Multi-walled carbon nanotubes increase anxiety levels in rats and reduce exploratory activity in the open field test. Dokl Biol Sci 464: 223-225, 2015.

13. Sandini TM, Udo MS, Reis-Silva TM, Sanches D, Bernardi MM, Flório JC and Spinosa Hde S: Prenatal exposure to integerrimine $\mathrm{N}$-oxide enriched butanolic residue from Senecio brasiliensis affects behavior and striatal neurotransmitter levels of rats in adulthood. Int J Dev Neurosci 47: $157-164,2015$

14. Menachem A, Chapman J, Deri Y, Pick CG and Katzav A: Immunoglobulin-mediated neuro-cognitive impairment: New data and a comprehensive review. Clin Rev Allergy Immunol 45: 248-255, 2013.

15. Grigor'ev NR, Batalova TA, Kirichenko EF, Sergievich AA and Cherbikova GE: Typological features in the behavior of rats. Neurosci Behav Physiol 38: 597-603, 2008.

16. Buslovich Slu, Kotelenets AI and Fridliand RM: An integral method of evaluating the open-field behavior of white rats. Zh Vyssh Nerv Deiat Im I P Pavlova 39: 168-171, 1989 (In Russian).

17. Pellow S, Chopin P, File SE and Briley M: Validation of open: Closed arm entries in an elevated plus-maze as a measure of anxiety in the rat. J Neurosci Methods 14: 149-167, 1985.
18. Pellow S and File SE: Anxiolytic and anxiogenic drug effects on exploratory activity in an elevated plus-maze: A novel test of anxiety in the rat. Pharmacol Biochem Behav 24: 525-529, 1986.

19. Grigor'ev NR: A method for the quantitative evaluation of search activity and refusal to search in an experiment with rats. Zh Vyssh Nerv Deiat Im I P Pavlova 46: 400-405, 1996 (In Russian).

20. Golokhvast K, Sergievich A and Grigoriev N: Geophagy (rock eating), experimental stress and cognitive idiosyncrasy. Asian Pac J Trop Biomed 4: 362-366, 2014.

21. Bianco A, Kostarelos K and Prato M: Applications of carbon nanotubes in drug delivery. Curr Opin Chem Biol 9: 674-679, 2005.

22. Mudie DM, Amidon GL and Amidon GE: Physiological parameters for oral delivery and in vitro testing. Mol Pharm 7: $1388-1405,2010$

23. Win-Shwe TT and Fujimaki H: Nanoparticles and neurotoxicity. Int J Mol Sci 12: 6267-6280, 2011.

24. Rosenblat JD, Brietzke E, Mansur RB, Maruschak NA, Lee Y and McIntyre RS: Inflammation as a neurobiological substrate of cognitive impairment in bipolar disorder: Evidence, pathophysiology and treatment implications. J Affect Disord 188: 149-159, 2015.

25. Grigor'ev NR, Li Tingli and Shao-Iuan' L: Cognitive abilities of rats in behavior of the active avoidance in age differences. $\mathrm{Zh}$ Vyssh Nerv Deiat Im I P Pavlova 64: 314-323, 2014 (In Russian). 